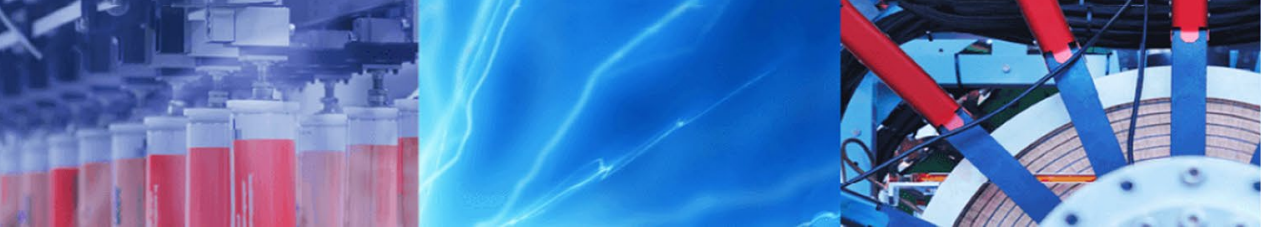

Research Article

\title{
Impact of binder as a formulation variable on the material and tableting properties of developed co-processed excipients
}

\author{
Yonni Eshovo Apeji ${ }^{1,2} \cdot$ Olubunmi Jumoke Olayemi $^{3} \cdot$ Sophie Nock Anyebe ${ }^{2} \cdot$ Chinyere Oparaeche $^{5,6}$. \\ Oluwaseun Adenike Orugun ${ }^{2,4} \cdot$ Adeniji Kehinde Olowosulu $^{2} \cdot$ Avosuahi Rukayat Oyi $^{2}$
}

(c) Springer Nature Switzerland AG 2019

\begin{abstract}
The functionality of a co-processed excipient is usually derived from its composition and the proportion of each excipient that is incorporated to yield a composite excipient. The aim of this study was to assess the impact of binder as a formulation variable on the material and tableting properties of developed co-processed excipients containing gelatin (SGS) and microcrystalline cellulose (SMS) as binders respectively in the same proportion. Two co-processed excipients, SGS and SMS were generated by combining tapioca starch (90\%), gelatin or microcrystalline cellulose $(7.5 \%)$ and colloidal silicon dioxide (2.5\%) using the co-fusion method. Particle size analysis and morphological assessment were carried out by light microscopy and scanning electron microscopy (SEM) respectively. DSC analysis was performed to evaluate the thermal behaviour of both materials and flow properties were assessed by measuring parameters like angle of repose, bulk and tapped densities, Carr's index and Hausner's ratio. Compaction behaviour of both materials was determined using Heckel and Walker equations and the compressibility-tabletability-compactibility (CTC) profile for each material was obtained. Particulate and bulk-level properties of SGS and SMS revealed spherical-shaped, free-flowing powders characterized by a glass transition event typical of amorphous polymers. Compaction analysis demonstrated greater degree of plastic deformation with SMS resulting in better tableting properties with respect to tensile strength and disintegration time. The outcome of the study shows that the choice of binder used in the formulation of a co-processed excipient plays a crucial role in defining the material and tableting properties of the co-processed excipient.
\end{abstract}

Keywords Binder - Gelatin $\cdot$ Microcrystalline cellulose $\cdot$ Co-processed excipients

\section{Introduction}

The success of a tableting operation requires the inclusion of functional excipients to the formulation. Excipients play the traditional role of ensuring that active pharmaceutical ingredients are processed into suitable dosage forms that will deliver the drug to the intended site of action after administration. Hence, the manufacturability, stability, bioavailability and efficacy of the drug are influenced by the presence of excipients in a formulation. The necessity for excipients with improved functionality has driven the research in the area of novel excipient development to increase the range of excipients that are available for pharmaceutical development.

Many of these researches have led to the commercialization of novel excipients like Prosolv ${ }^{\circledast}$ (JRS Pharma),

\footnotetext{
$\triangle$ Yonni Eshovo Apeji, yehonathanapeji@gmail.com; yeapeji@abu.edu.ng| ${ }^{1}$ Department of Pharmaceutics, National Institute of Pharmaceutical Education and Research (NIPER), S.A.S. Nagar, 160062 Punjab, India. ${ }^{2}$ Department of Pharmaceutics and Pharmaceutical Microbiology, Faculty of Pharmaceutical Sciences, Ahmadu Bello University, Zaria, Nigeria. ${ }^{3}$ Department of Pharmaceutical Technology and Raw Materials Research, National Institute for Pharmaceutical Research and Development (NIPRD), Idu, Abuja, Nigeria. ${ }^{4}$ Department of Pharmaceutics and Pharmaceutical Microbiology, Faculty of Pharmaceutical Sciences, Kaduna State University, Kaduna, Kaduna State, Nigeria. ${ }^{5}$ Gamlen Tableting Ltd, London, UK. ${ }^{6}$ Department of Pharmaceutical, Chemical and Environmental Sciences, Faculty of Engineering and Science, University of Greenwich, London, UK.
} 
Ludipress $^{\circledast}$ (BASF) and StarLac ${ }^{\circledast}$ (Roquette Pharma). Currently, attention is being paid to direct compression (DC) as the preferred method of tableting because it addresses most of the limitations encountered with the conventional method of wet granulation. This has steered the development of novel excipients greatly towards those that possess direct compression functionality. Only a few single excipients exist with direct compression functionality and on the top of the list is microcrystalline cellulose (MCC) which has been classified as a dry binder in the formulation of tablets by dry granulation and direct compression [1]. All other excipients that are employed in direct compression tableting are a combination of two or more excipients known as co-processed excipients. Co-processing is a particle engineering technique that facilitates the development of a composite excipient with improved functionality [2-4]. The performance attributes of a co-processed excipient are usually determined by the composition of the co-processed excipient with respect to the primary excipients used as well as the proportion of each primary excipient in the co-processed excipient $[3,5]$. Research into co-processing of excipients has yielded products with diverse functionalities and performance attributes. These functionalities include orally disintegrating ability $[6,7]$, improved dilution potential [8], rapid disintegration [9], enhanced direct compression functionality [10], multifunctional excipient $[4,8]$, improved tabletability [11], and improved dispersibility [12].

Binders are important materials included in the formulation of tablets to facilitate bonding of powder particles during granulation. They ensure the agglomeration and cohesion of the granules to each other, promoting appropriate compactibility and free-flowing properties [13]. Depending on their mode of incorporation in tablet formulation, binders are classified as solution binders (hydroxypropyl methylcellulose, gelatin and polyvinylpyrrolidone) or dry binders (microcrystalline cellulose). Binders have been known to modulate the compressibility of a tablet formulation and so has become a candidate material in co-processing to improve the functionality of excipients with poor compressibility. To overcome the relative poor compactibility of lactose, co-processed excipient based on the combination of a-lactose monohydrate (a brittle material) and plastic binders (microcrystalline cellulose and polyvinyl pyrrolidone) have been developed with considerable success [10]. Similarly, a study carried out by Wang et al. (2015) demonstrated an improvement in the tabletability of lactose when co-processed with hydroxypropyl methylcellulose (HPMC E3) and crospovidone (PVPP). Other studies related to improving the direct compression potential of starches has been achieved by co-processing with binders like acacia $[14,15]$. In the present study, two co-processed excipients composed of tapioca starch and colloidal silicon dioxide but differing in the binder content were generated by co-fusion. The aim of the study was to assess the impact of binders differing in their mode of incorporation on the material and tableting properties of the generated co-processed excipients. Gelatin was selected as an example of a solution binder while microcrystalline cellulose was chosen as a dry binder. Both binders were incorporated in the same proportion in their respective formulations.

\section{Materials and methods}

Tapioca starch was obtained as a gift sample from Quality Starch and Chemicals (Tamil Nadu, India), Microcrystalline cellulose (Avicel PH 112, FMC Biopolymer, Philadelphia, PA), Gelatin (May and Baker Ltd, England) and colloidal silicon dioxide (Cab-o-Sil ${ }^{\circledR}$, Cabot $\mathrm{GmbH}$, Germany). All other materials used were of analytical grade.

\subsection{Preparation of co-processed excipients (SGS and SMS)}

Co-processing of excipients was carried out using the method described by Adeoye and Alebiowu [16] with modifications. A $40 \% \mathrm{w} / \mathrm{w}$ suspension of tapioca starch (TS) was prepared by dispersing $90 \mathrm{~g}$ of tapioca starch (TS) in $135 \mathrm{~mL}$ distilled water. Gelatin $(7.5 \mathrm{~g})$ and colloidal silicon dioxide $(2.5 \mathrm{~g}$ ) were added to the suspension and stirred for $5 \mathrm{~min}$. Further mixing was continued on a shaker water bath (Julabo SW 23, Seelbach, Germany) set at $54 \pm 2{ }^{\circ} \mathrm{C} / 60 \mathrm{rpm}$ for $15 \mathrm{~min}$. The mixture was allowed to equilibrate at $25 \pm 2{ }^{\circ} \mathrm{C}$ and homogenized at a speed of 11,000 rpm (Ultra Turrax T 25 basic Ika ${ }^{\circledR}$ Werke, India) for $5 \mathrm{~min}$, it was then dried partially at $25 \pm 2{ }^{\circ} \mathrm{C}$ for $2 \mathrm{~h}$ before being finally dried in the Fluid bed dryer (Retsch TG 100, Germany) at $40^{\circ} \mathrm{C}$ for $10 \mathrm{~min}$. The co-processed excipient (SGS) obtained was screened through a sieve $(500 \mu \mathrm{m})$ and stored in an air-tight container kept in a desiccator containing phosphorous pentoxide $\left(\mathrm{P}_{2} \mathrm{O}_{5}\right)$ prior to characterization studies. The entire process was repeated to prepare SMS containing microcrystalline cellulose (MCC) as binder.

\subsection{Particle size analysis}

Particle size analysis was carried out for SGS and SMS using a Leica DMLP light microscope (Leica, Microsystems Wetzlar GmbH, Wetzlar, Germany). The diameter corresponding to the length along the longest axis was measured for each particle and a minimum of 500 particles were counted for each material. Particle size was expressed as $d_{10}, d_{50}$, and $d_{90}$ corresponding to 10,50 and $90 \%$ of cumulative undersize particles respectively. 


\subsection{Scanning electron microscopy (SEM)}

The shape and surface morphology of both materials were examined using a scanning electron microscope (S-3400, Hitachi Ltd., Tokyo, Japan) operated at an excitation voltage of $15 \mathrm{kV}$. The samples were prepared by mounting on a steel stage using a double-sided adhesive tape and sputter-coated with gold prior to image capture at various magnifications.

\subsection{Differential scanning calorimetry}

Thermal analysis of the materials was evaluated using DSC, Model Q2000 (TA Instruments, New Castle, DE USA). Prior to analysis, the DSC instrument was calibrated using high purity indium standard and continuously purged with dry nitrogen flowing at $50 \mathrm{ml} / \mathrm{min}$. Powder samples weighing about $5 \mathrm{mg}$ each of SMS and SGS were heated in standard Tzero aluminium pans at $20^{\circ} \mathrm{C} / \mathrm{min}$ from 25 to $300^{\circ} \mathrm{C}$. Data analysis was performed using Universal ${ }^{\circledR}$ Analysis 2000 (version 4.5A) software. Modulated differential scanning calorimetry (MDSC) scan was carried out to obtain a better resolution of the glass transition event from the reversing heat flow signal.

\subsection{Angle of repose}

Angle of repose of both powders was measured using the granulate flow tester (GTB, Erweka, Germany). The powder sample ( $20 \mathrm{~g}$ ) was poured at $45^{\circ}$ into a stainless-steel funnel and allowed to flow out through the orifice measuring $10 \mathrm{~mm}$ in diameter unto a flat surface. The height of the conical heap of powder was measured automatically using an integrated driven laser system and the angle of repose calculated using the formula, $\theta=\operatorname{Tan}^{-1} \mathrm{~h} / \mathrm{r}$. A mean of three replicates was recorded as the angle of repose.

\subsection{Bulk, tapped and true densities}

The volume occupied by $20 \mathrm{~g}$ powder of each sample poured into a $100 \mathrm{~mL}$ measuring cylinder of a tapped density tester (USP tap density apparatus, Electro lab, Mumbai, India) was recorded as the bulk volume. The tapped volume was obtained by tapping the contents of the measuring cylinder to constant volume according to the USP II method. Bulk and tapped volumes were obtained in triplicate and were used to calculate the bulk and tapped densities. Hausner ratio (HR) and Carr's index $(\mathrm{Cl})$ were calculated for each sample using the bulk and tapped densities and the results were expressed as mean \pm standard deviation.

True density $\left(\rho_{T}\right)$ of both materials was determined by the gas displacement technique using the Helium
Pycnometer (Pycno 30, Smart Instruments, India). Measurements were taken in triplicate at $25 \pm 2{ }^{\circ} \mathrm{C} / 40 \pm 2 \% \mathrm{RH}$ and the mean \pm standard deviation was recorded.

\subsection{Compaction studies}

The compaction behaviour of both powders was evaluated using out-of-die analysis. Tablets weighing $400 \mathrm{mg}$ were compressed individually at pressures ranging from 35-300 MPa on a Hydraulic Carver Press (Model 3912, Carver Inc., USA) using $13 \mathrm{~mm}$ flat-faced punches. Prior to compression, the die cavity was lubricated with a dispersion of magnesium stearate in ethanol. The compacts were held for $30 \mathrm{~s}$ at maximum pressure during the last stage of compression leading to detachment and ejection of the tablet. After ejection, the tablets were kept in a desiccator for $24 \mathrm{~h}$ to allow for elastic recovery. The tablet parameters of weight, thickness and crushing strength were measured for each tablet and the following equations (Eqs. 1, 2, 3,4 and 5 ) were used to calculate volume (V), apparent density $\left(\rho_{A}\right)$, relative density $(D)$, porosity $(\varepsilon)$ and tensile strength (TS) respectively.

$V=\pi r^{2} h$

$\rho_{A}=\frac{W}{\pi r^{2} h}$

$D=\frac{\rho_{A}}{\rho_{T}}$

$\varepsilon=1-D$

$T S=\frac{2 F}{\pi d t}$

where $r$ corresponds to the radius of the die, $h$ is the thickness of the tablet, $W$ is the weight of the tablet, $F$ is the crushing strength and $d$ is the diameter of the die.

The parameters obtained above were fitted into Heckel [17] and modified Walker [18, 19] equations (Eqs. 6 \& 7) respectively to generate the corresponding compaction plots.

$\ln \left(\frac{1}{1-D}\right)=K P+A$

$V^{\prime}=-W^{\prime} \log P+V_{S P^{\prime}}$

The compressibility plot (porosity against compaction pressure), compactibility plot (Tensile strength against porosity) and tabletability plot (tensile strength against compaction pressure) were also generated for each material. 


\subsection{Tableting}

Tablets (400 mg) were prepared by compressing directly a powder blend of ibuprofen and the co-processed excipient (SGS or SMS) (1:1) on a Rimek rotary tablet press (Mini II D, Karnavati Engineering Pvt. Ltd., India) fitted with flat-faced $12-\mathrm{mm}$ punch and die tooling. The compaction force was kept constant for both batches of tablets. The tablets were kept for $24 \mathrm{~h}$ after compression to allow for elastic recovery before evaluation of tablet properties. The following properties of tablets were evaluated according to BP specifications [20].

\subsection{Weight uniformity}

The weight of twenty randomly selected tablets from each batch of tablets was obtained using an analytical scale (Mettler-Toledo AG 285, Switzerland) and the mean and standard deviation of each sample was recorded.

\subsection{Tablet thickness}

The thickness of ten tablets chosen at random from each batch was measured using a digital micrometre screw gauge (Mitutoyo, Japan) and a mean of ten replicates was recorded with standard deviation.

\subsection{Crushing strength}

The breaking force required to crush a tablet along its diameter was determined using a tablet hardness tester $(\mathrm{TBH} 20$, Erweka, Germany). An average of six replicates was obtained for each batch and the tensile strength (Ts) was computed using the equation,

$T s=\frac{2 F}{\pi d t}$

where $\mathrm{F}$ is the crushing strength $(\mathrm{N}), d$ and $t$ are diameter and thickness respectively.

\subsection{Friability}

Tablet friability was evaluated using a Friabilator (EF-2, Electro lab, India). Ten tablets were weighed initially, placed in the friabilator and allowed to rotate at $25 \mathrm{rpm}$ for $4 \mathrm{~min}$. The tablets were re-dusted, re-weighed and the difference in tablet weight was determined. Friability was calculated as follows:

$\%$ friability $=\frac{W_{i}-W_{f}}{W_{i}} \times 100$ where $W_{i}$ and $W_{f}$ are the initial and final weights of tablets respectively.

\subsection{Disintegration time}

The disintegration time of six tablets sampled at random for each batch was determined using a disintegration apparatus (ED-2L, Electro lab, India) as described in the British Pharmacopoeia [20].

\subsection{Determination of disintegration efficiency ratio (DER)}

The disintegration efficiency ratio (DER) was determined using the relationship,

$D E R=\frac{C_{S} / F_{R}}{D_{T}}$

where $C_{S}, F_{R}$ and $D_{T}$ are crushing strength, friability and disintegration time respectively.

\subsection{In vitro dissolution studies}

In vitro dissolution studies were conducted using the dissolution tester (USP) (TDT-08L, Electro lab, India). The USP Apparatus II (paddle) method was adopted as recommended by USP [21]. Phosphate buffer (pH 7.2; $900 \mathrm{~mL})$ was prepared and used as the dissolution medium. One tablet from each formulation was dropped into the dissolution medium $\left(37 \pm 0.5^{\circ} \mathrm{C}\right)$ and the apparatus set to rotate at $50 \mathrm{rpm}$ for $60 \mathrm{~min}$. Aliquots of $5 \mathrm{~mL}$ were withdrawn at specific time intervals and replaced with equal volume of phosphate buffer ( $\mathrm{pH}$ 7.2) maintained the same temperature as that of the dissolution medium. Each withdrawn sample was filtered using a $0.2 \mu$ membrane disc filter and suitably diluted with phosphate buffer $(\mathrm{pH}$ 7.2). The diluted samples were assayed for ibuprofen by reading the absorbance at $221 \mathrm{~nm}$ using the UV/VIS spectrophotometer (Lambda 35, PerkinElmer, USA) and the amount of drug released was calculated using the regression data equation $\left(y=0.0951 x, R^{2}=0.9981\right)$ obtained from the calibration curve of ibuprofen. The dissolution profile of ibuprofen was determined by plotting a graph of percentage drug released against time.

\subsection{Statistical analysis}

Data analysis was carried out in Microsoft Excel using the analytical tool, ANOVA: Single factor to evaluate the differences in tableting properties of both materials. Differences in tableting properties of both materials were considered significant at $p \leq 0.05$. 


\section{Results}

Particle size analysis of the two co-processed excipients is presented in Table 1. Comparing the $d_{10}, d_{50}$ and $d_{90}$ parameters, particle size of SGS was approximately twice as large as that of SMS. A narrow particle size distribution was obtained for SMS due to its lower span value of 1.26.

Figure $1 \mathrm{a}, \mathrm{b}$ displays the SEM pictures of SGS and SMS respectively. Both materials are characterized by sphericalshaped particles with several particles showing a deviation from sphericity. Surface morphology reveals that the SGS particles appear smoother than those of SMS. The images of SGS and SMS reveals a homogeneous dispersion of the interacting excipients resulting in the formation of a single composite excipient with minimal appearance of discrete primary particles of the individual excipients.

Thermal analysis of SGS and SMS represented by the DSC curve in Fig. 2 shows a characteristic glass transition event occurring in the reversing heat flow signal for both materials. The onset of glass transition was slightly lower for SGS occurring at a temperature of $251.53^{\circ} \mathrm{C}$ compared to $254.02{ }^{\circ} \mathrm{C}$ obtained for SMS.

The powder properties of SGS and SMS are presented in Table 2. Both powders demonstrated good flow behaviour on account of the low values obtained for $\mathrm{AR}, \mathrm{Cl}$ and $\mathrm{HR}$.

Table 1 Particle size analysis of SGS and SMS using microscopy

\begin{tabular}{lllll}
\hline Material & $D_{10}(\mu)$ & $D_{50}(\mu)$ & $D_{90}(\mu)$ & Span \\
\hline SGS & 77.5 & 145 & 289 & 1.46 \\
SMS & 44 & 75.5 & 139.5 & 1.26 \\
\hline
\end{tabular}

SGS Co-processed excipient containing gelatin as binder

SMS Co-processed excipient containing microcrystalline cellulose as binder
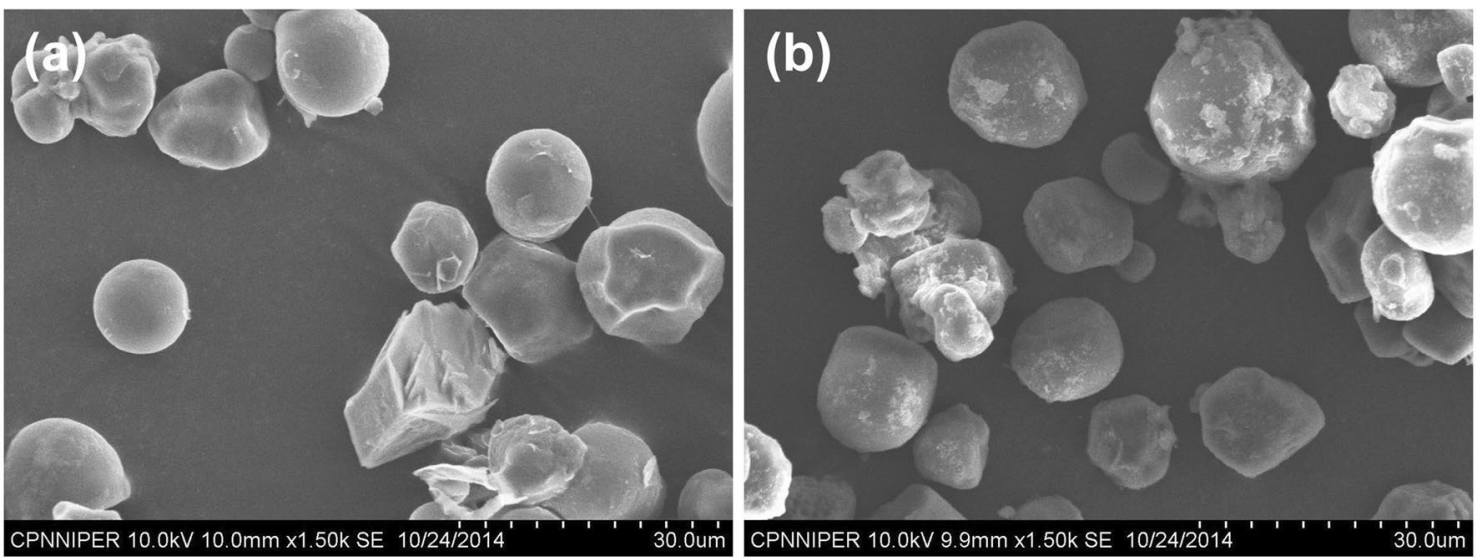

The BD and TD obtained for SMS was greater than that of SGS indicating a greater degree of packing in the powder bed.

The true density of both powders did not differ significantly although SGS was observed to have a slightly higher value of $1.45 \mathrm{~g} / \mathrm{cm}^{3}$ than that of SMS. There was, however, a remarkable difference in their swelling index (SI) as SMS was found to swell about 4 times more than SGS.

Table 3 is a summary of the compaction parameters derived from Heckel and Walker analysis (Fig. 3). The mean yield pressure $\left(P_{\gamma}\right)$ resolved as the inverse of the slope of the linear portion of the Heckel plot was found to be lower for SMS compared to SGS indicating a higher degree of plasticity. The total degree of densification of the powder bed at the early stages of compression was quantified as the $D_{A}$ parameter. The extent of densification occurring as a result of particle rearrangement $\left(D_{A}\right)$ was seen to be higher for SGS (0.745) than that of SMS (0.614). Densification attributed to particle motion and filling of the die at low pressures $\left(D_{0}\right)$ on the other hand, was higher for SMS compared to SGS. This is consistent with the values of bulk and tapped densities obtained for SMS which was significantly higher than that of SGS. However, densification contributed by particle fragmentation at low pressures $\left(D_{B}\right)$ was higher for SGS in relation to SMS indicating that lower amount of plastic deformation occurred during compression. This agrees with the higher yield pressure obtained for SGS. The Walker's $W^{\prime}$ coefficient of compressibility indicates that SMS is more compressible than SGS owing to the higher value obtained as compressibility coefficient $\left(W^{\prime}\right)$ for SMS (Table 3).

The compaction behaviour of the two materials was also evaluated using the compressibility-tabletabilitycompactibility (CTC) profile presented in Fig. $4 a-c$. The compressibility plot (Fig. 4a) relates the effect of compression pressure on porosity and shows a gradual decline in

Fig. 1 SEM images of $\mathbf{a}$ SGS and $\mathbf{b}$ SMS 


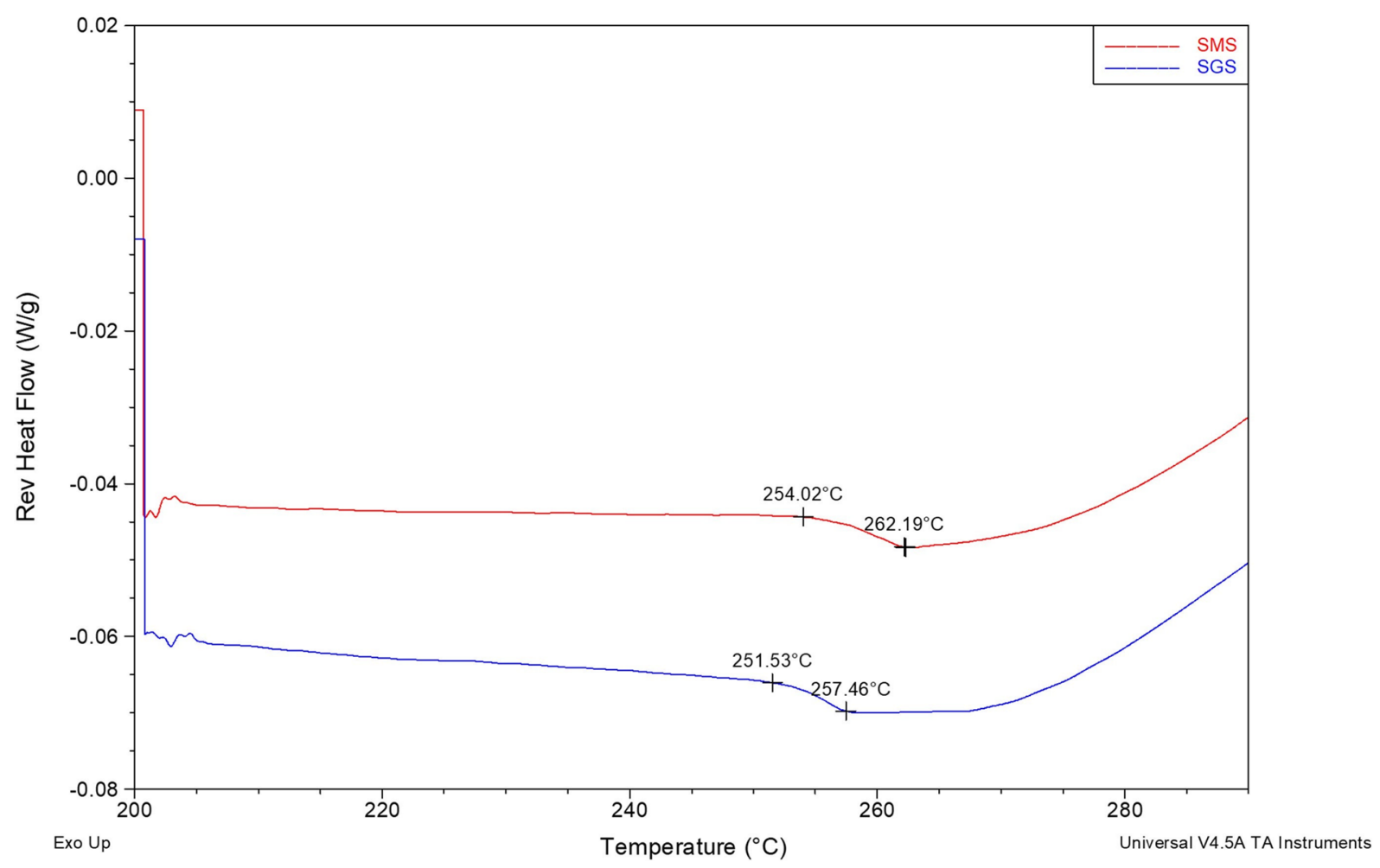

Fig. 2 DSC overlay of SGS and SMS

Table 2 Powder properties of SGS and SMS

\begin{tabular}{llllllll}
\hline Material & $\mathrm{AR}\left({ }^{\circ}\right)$ & $\mathrm{BD}(\mathrm{g} / \mathrm{mL})$ & $\mathrm{TD}(\mathrm{g} / \mathrm{mL})$ & $\mathrm{PD}(\mathrm{g} / \mathrm{mL})$ & $\mathrm{Cl}(\%)$ & $\mathrm{HR}$ & $\mathrm{SI}(\%)$ \\
\hline $\mathrm{SGS}$ & $21.6(0.3)$ & $0.48(0.01)$ & $0.57(0.02)$ & $1.45(0.02)$ & $14.4(4.53)$ & $1.17(0.06)$ & $10.15(1.49)$ \\
SMS & $22.1(0.2)$ & $0.54(0.02)$ & $0.67(0)$ & $1.43(0.03)$ & $19.6(2.53)$ & $1.24(0.04)$ & $45.69(1.94)$ \\
\hline
\end{tabular}

$A R$ angle of repose; $B D$ bulk density; $T D$ tapped density; $P D$ particle (true) density; $C I$ Carr's index; $H R$ Hausner's ratio; SI Swelling index

Table 3 Heckel and Walker parameters for SGS and SMS

\begin{tabular}{llllll}
\hline Material & $P_{y}$ & $D_{A}$ & $D_{0}$ & $D_{B}$ & $W^{\prime}$ \\
\hline SGS & 200 & 0.745 & 0.393 & 0.352 & 29.64 \\
SMS & 128.21 & 0.614 & 0.469 & 0.145 & 41.04 \\
\hline
\end{tabular}

porosity for both materials as the compression pressure increases. Lower levels of porosity were obtained for SGS at pressures ranging from 35-150 MPa but beyond $150 \mathrm{MPa}$, lower levels were obtained for SMS corresponding to greater compressibility at higher pressures.

The compactibility plot (Fig. 4b) shows the relationship between tensile strength and porosity of compacts. For both materials, tensile strength increased with decreasing porosity. The tensile strength of SGS compacts were higher at all porosities (0.1-0.4). At porosities below 0.1, tensile strength values of SMS were observed to be higher than those of SGS. Compacts of SMS produced the lowest porosity value at maximum compaction pressure. The tabletability profile for both materials represented by a plot of tensile strength against compression pressure (Fig. 4c) shows that tensile strength increased as the compression pressure increased. Higher tensile strength values were obtained with SGS at low and intermediate pressures. However, a maximum limit was reached for SGS at $200 \mathrm{MPa}$ while the tensile strength of SMS continued to rise with increasing pressure beyond $200 \mathrm{MPa}$.

The tableting properties of SGS and SMS are summarized in Table 4. The mean weight and thickness parameters of tablets for both materials were similar. There were significant differences in the crushing strength, tensile strength and disintegration time of tablets from both materials at $p<0.05$. It was observed that SMS produced stronger tablets that disintegrated faster than those of SGS. 

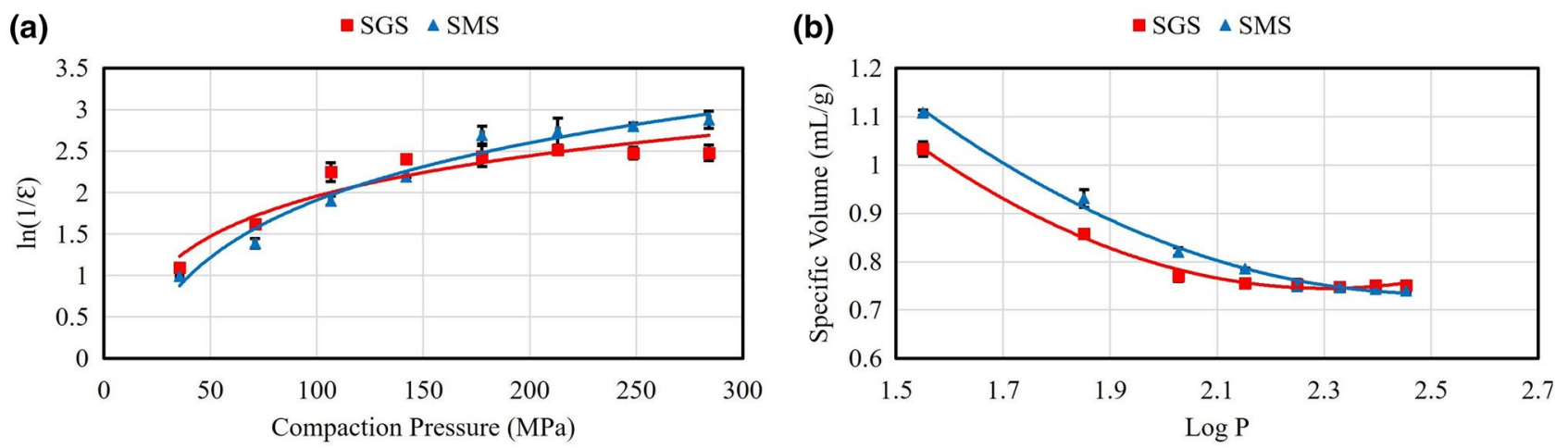

Fig. 3 a Heckel and $\mathbf{b}$ Walker plots of SGS and SMS
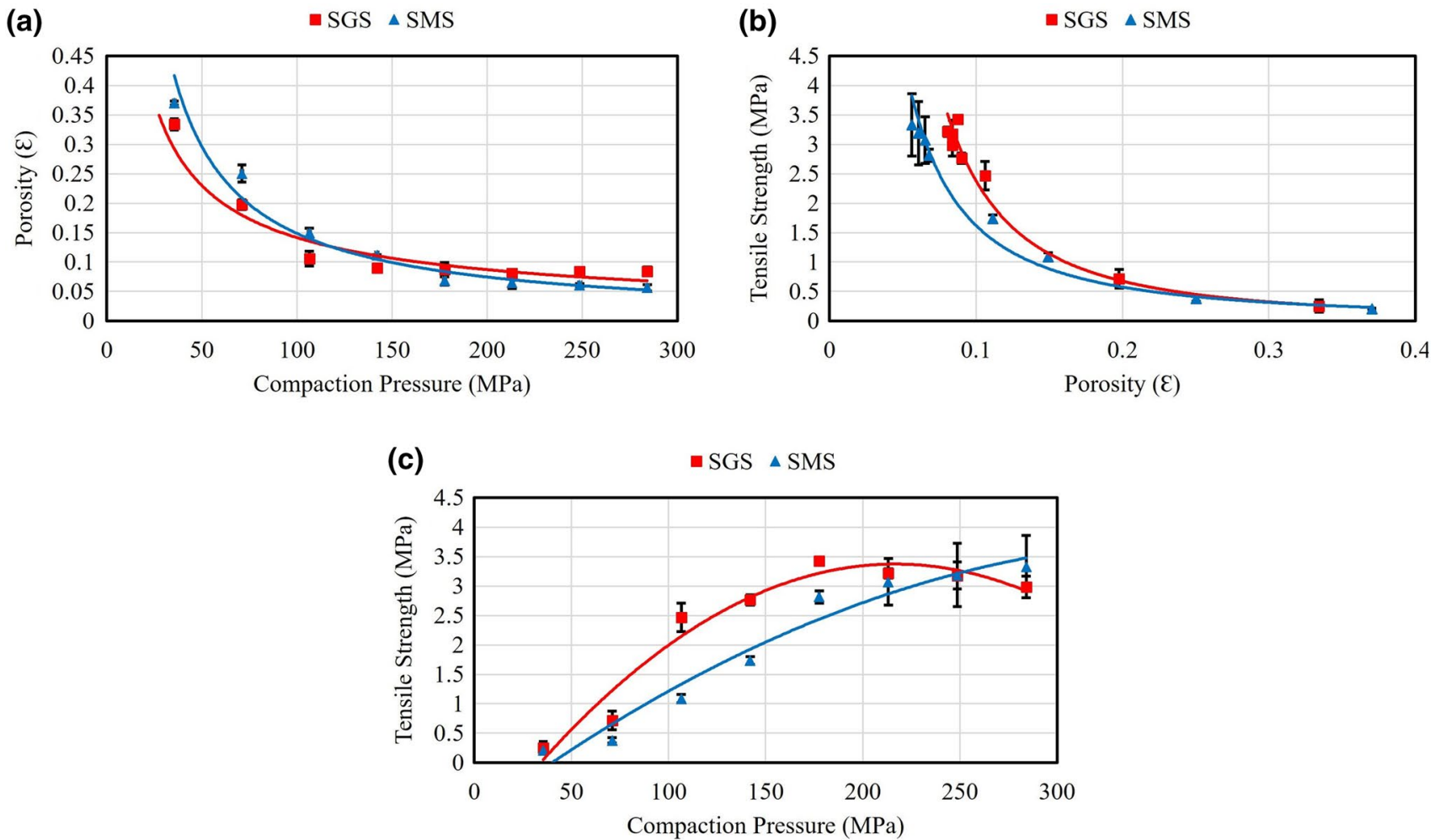

Fig. 4 CTC plots of SGS and SMS. a Compressibility plot, b Compactibility plot and c Tabletability plot

Table 4 Physical properties of tablets prepared using SGS and SMS as DC excipients

\begin{tabular}{lllllllll}
\hline Material & Weight $(\mathrm{mg})$ & Thickness $(\mathrm{mm})$ & $\mathrm{CS}(\mathrm{N})$ & $\mathrm{TS}(\mathrm{MPa})$ & $\mathrm{DT}(\mathrm{min})$ & $\mathrm{FR}(\%)$ & $\mathrm{DER}$ & $\mathrm{T}_{50 \%}(\mathrm{~min})$ \\
\hline SGS & $399.7(4.22)$ & $3.43(0.03)$ & $79.38(7.7)$ & $1.23(0.12)$ & $0.32(0.04)$ & $0.57(0.07)$ & 435.20 & 5 \\
SMS & $399.4(6.81)$ & $3.43(0.05)$ & $91.5(4.04)$ & $1.42(0.06)$ & $0.16(0.03)$ & $0.59(0.04)$ & 969.28 & 4 \\
\hline
\end{tabular}

CS Crushing strength; TS Tensile strength; DT Disintegration time; FR Friability; DER Disintegration efficiency ratio; $T_{50 \%}$ Time taken to attain $50 \%$ drug release 
The DER for both materials shows that SMS had a much higher value implying a better balance of disintegrating and binding properties. Friability did not differ widely for both materials and the time taken to attain $50 \%$ of drug release was faster with SMS compared to SGS. The dissolution plot (Fig. 5) shows a similar release pattern for both materials.

\section{Discussion}

The principal objective of co-processing is to engineer the functionality of existing excipients for a desired application in solid dosage form development. It is necessary therefore to make rational selection of the interacting excipients that will encapsulate the desired attributes in the composite excipient $[3,22]$. In our study, gelatin (GEL) and microcrystalline cellulose (MCC) were chosen as models of wet and dry binders respectively in the preparation of SGS and SMS. One key attribute of co-processed excipients that is modulated during development is the particle size $[23,24]$ which contributes significantly to the functionality of the co-processed excipient $[8,11]$. Bulk properties of flow and compressibility has been enhanced as a result of particle size modification $[25,26]$. The difference in particle size observed for SGS and SMS can be attributed to the type and mode of incorporation of the binder during co-processing. Gelatin has been used traditionally as a binding agent in the formulation of tablets by wet granulation [27]. Hence, its propensity to increase particle size significantly was higher due to its agglomerating effect resulting in the cohesion of powder particles by the formation of liquid bridges which led to particle size enlargement [28]. On the other hand, microcrystalline cellulose does not exert that agglomerating effect that leads to increase in particle size because of its inability to form liquid bridges during processing [1]. As a result, particles of SGS were significantly larger in size than those of SMS. This suggests that the type of binder incorporated in the formulation of co-processed excipients influences to a reasonable degree the particle size of the composite excipient generated.

Co-processing of excipients did not alter significantly the particle shape of the parent excipient, tapioca starch as the particles of SGS and SMS maintained the characteristic spherical shape of tapioca starch $[29,30]$. The percentage proportion of the binders (7.5\%) employed in this study may not have been sufficient enough to cause a deviation in the sphericity of tapioca starch considering that the particle shapes of gelatin and microcrystalline cellulose are irregular and fibrous respectively $[5,31]$. However, there seems to be slight modifications of the surface morphology of SMS as the particles appeared to have what looked like deposits on its surface. This may be ascribed to the presence of colloidal silicon dioxide (CSD) which remained largely on the surface of SMS particles as opposed to the integration of CSD into the particle structure of SGS as a result of the binding effect of gelatin (Fig. 1). The deposits of CSD on the surface of SMS particles may be responsible for the superior swelling action and rapid disintegration time exhibited by SMS. This concurs with the findings of Rojas and Kumar (2011) who related the rapid disintegrating action of silicified microcrystalline cellulose II to the deposition of CSD on the surface of the co-processed excipient resulting in rapid ingress of water and subsequent disintegration ( $<2 \mathrm{~min}$ ). Particle shape has been implicated in the bulk flow of powders as spherical shaped particles tend to flow better due to limited interparticulate friction [32]. The flow properties of SMS and SGS were observed to be excellent flow with respect to the indices
Fig. 5 In vitro drug-release profile of SGS and SMS

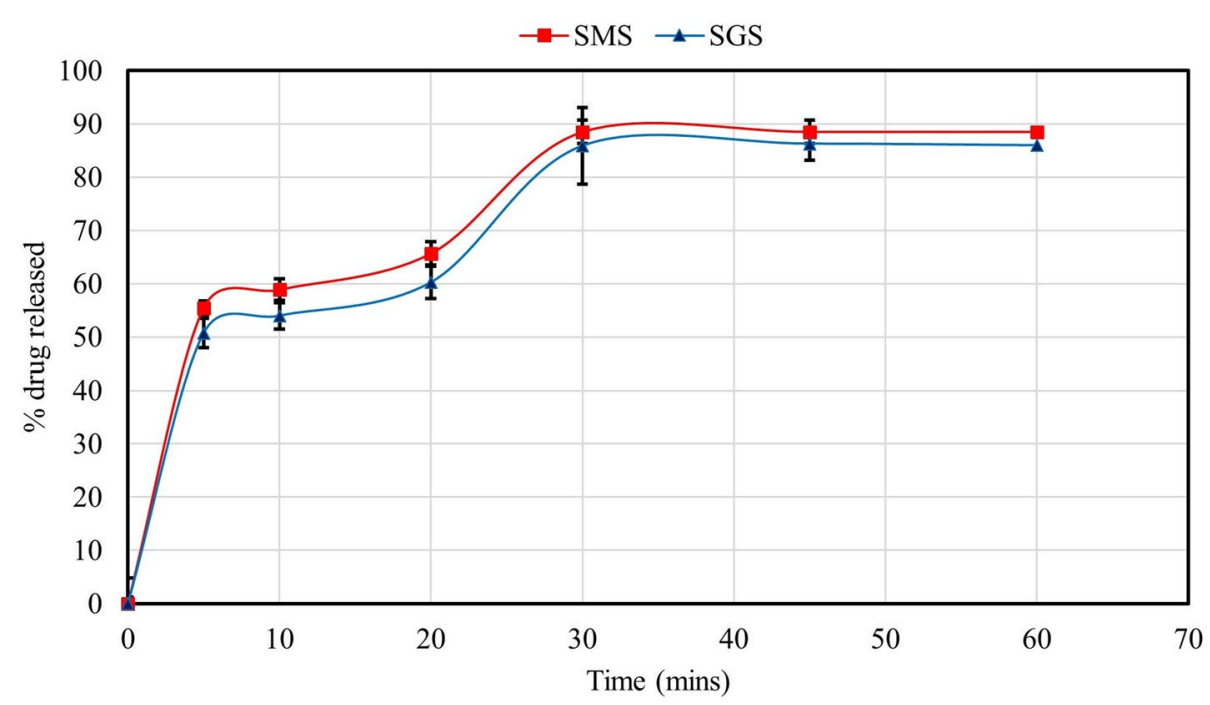


of angle of repose, Carr's index and Hausner's ratio and this is consistent with their spherical particle shape.

The appearance of a base-line shift in the DSC curves of SGS and SMS corresponds to a glass transition (Tg) event (Fig. 2) and this implies that both materials are predominantly amorphous. Amorphous or glassy polymers do not generally display a sharp melting point; instead, they soften over a wide temperature range. The difference in Tg obtained for both materials though not significant can be attributed to the difference in composition of the coprocessed excipients. A lower Tg was obtained for SGS possibly because gelatin degrades at temperatures exceeding $40^{\circ} \mathrm{C}$ [33] while microcrystalline cellulose degrades at temperatures exceeding $100^{\circ} \mathrm{C}$. Glass transition temperature $(\mathrm{Tg})$ for linear organic polymers range from -100 to $\geq 300^{\circ} \mathrm{C}$ [34]. From a pharmaceutical standpoint, $\mathrm{Tg}$ is an important factor for solid dosage forms. For solid dosage forms, amorphous polymers are expected to remain solid at room temperature or at the storage temperature to ensure the stability of the product. The high $\mathrm{Tg}$ of both materials will guarantee the stability of products prepared using these materials as excipients. Furthermore, a less crystalline or amorphous polymer is preferred in solid dosage formulations because they facilitate the release of drugs compared to crystalline polymers that impede the release of drugs because they display barrier properties [34].

The flow properties exhibited by both materials confirm the efficacy of co-processing as a particle engineering technique in improving the flow functionality of excipients with poor flowability status. This is in agreement with several other studies carried out that demonstrated improvement in flowability as a result of co-processing $[4,7,24$, 35]. Improvement in powder flow could be attributed to the increase in particle size and the spherical shape recorded for both materials. The ability of powders to flow well during tableting is a necessary requirement for materials that are designed for direct compression because they exclude the processing step of granulation that imparts flowability to the powder blend. Most direct compression formulations usually consist of the excipient in higher proportions (60-95\%), hence the ability of this excipient to flow greatly influences the flow behaviour of the powder blend. Enhanced flow of the powder blend will result in tablets with uniform weight and content which conforms to quality attributes of robust tablets. The remarkably higher swelling index for SMS relative to SGS (Table 2) may be associated with the high intra-particulate porosity of microcrystalline cellulose which promotes swelling by means of capillary action [1] coupled with the deposition of CSD on the surface of SMS particles as shown by the SEM image (Fig. 1). On the other hand, the limited swelling profile of SGS could be linked to the formation of a non-porous gel by gelatin that prevents further uptake of water [5]. Swelling is an established mechanism by which some disintegrants exert their action [36]. Tablets containing SMS were found to disintegrate faster than those containing SGS thereby validating its superior swelling profile.

The ability of a material to form solid compacts or tablets is quantified by its mechanical properties [37]. Mechanical properties of a powder relates to its response to applied pressure during compression [38]. Powders under the effect of an external mechanical stress are subject to either elastic deformation, plastic deformation, viscoelastic deformation, or fragmentation as the main mechanism for deformation [39]. Plastic deformation has been recognized as the most important deformation mechanism that facilitates tablet formation [39]. According to Heckel analysis, materials having a relatively low yield pressure $\left(P_{Y}\right)$ undergo a greater degree of plastic deformation during compression. A lower yield pressure was obtained for SMS implying a higher degree of plasticity (Table 3). Plasticity has been associated with a material's ability to undergo irreversible deformation during compression that leads to the formation of permanent bonds that are retained after decompression and elastic recovery during tableting [40]. The low yield pressure recorded for SMS has been linked to the ability of microcrystalline cellulose to undergo plastic deformation during compression due to the presence of slip planes in its micro structure [1]. On the other hand, a higher degree of particle fragmentation $\left(D_{B}\right)$ was recorded for SGS which correlates with the higher yield pressure $\left(P_{Y}\right)$ obtained for the same material (Table 2). Materials that have high propensity for particle fragmentation are usually brittle and hard, thereby resisting compression leading to particle breakage and subsequent plastic deformation at higher yield pressures [41]. The higher yield pressure of SGS may be related to the brittle fracture tendency of gelatin [27]. Like the Heckel model, the Walker profile also provides information about the plasticity of a material [42]. The higher the absolute Walker coefficient $(W)$, the more readily the powder reduces its volume, and therefore the more compressible and plastic it is. The higher W' of SMS shows that it readily undergoes plastic deformation during compression compared to SGS. This is consistent with the findings of Heckel analysis.

Tabletability has been used as a performance indicator to study the compaction behaviour of pharmaceutical powders and is usually defined by the contributing factors of compressibility and compactibility [43]. Compressibility refers to the ability of a material to undergo volume reduction when pressure is applied and can be graphically illustrated as a porosity-pressure relationship [44]. The application of pressure to a powder bed in a confined space initiates particle rearrangement that leads to the filling of the pore spaces, deformation and reduction 
in volume thereby increasing the packability and density of the powder [38]. When this happens, particles are brought near each other thereby increasing bonding area. The bonding area-bonding strength model proposed by Sun (2011) provides the correlation to compressibility and compactibility. The minimal difference observed in the compressibility profile of both materials may be attributed to the deformation mechanism exhibited by both materials during compression. Lower porosity levels obtained at higher pressures for SMS demonstrates higher compressibility which is related to its greater degree of plastic deformation as seen in the Heckel analysis. With respect to tabletability, compressibility of the material contributes the bonding area over which inter-particulate bonds are generated thus higher compressibility and consequent larger bonding area was obtained for SMS relative to SGS. Compactibility is the ability of a material to form tablets of sufficient tensile strength under the effect of densification and is strongly correlated with the strength of interparticulate bonds in the compact (bonding strength). The bonding strength of each material increased with decreasing porosity (Fig. 4b); much lower levels of porosity $(<0.1)$ were obtained for SMS giving rise to compacts with higher tensile strength. The higher bonding strength observed with SMS may be linked to a stronger interfacial adhesion occurring during bonding [45]. In addition, the presence of microcrystalline cellulose in the particle structure of SMS could have facilitated the formation of hydrogen bonds between adjacent particles contributing to the higher tensile strength obtained for SMS compacts at lower porosity. Other factors that may have influenced the compactibility of both materials include properties like the particle size and shape [37]. Tabletability is basically known as the capacity of a powdered material to be transformed into a tablet of specified strength under the effect of compaction pressure [46]. The differences observed in the tabletability profiles of both materials reflected the differences in their compressibility and compactibility profiles. Hence, the tabletability of each material was governed by their respective compressibility and compactibility profiles. The continuous rise in tabletability of SMS even at pressures beyond $200 \mathrm{MPa}$ implies that bonding area and bonding strength increased with higher loads. Hence, there was reduced tendency for elastic recovery during decompression and after ejection. However, the tabletability of SGS was observed to plateau at $200 \mathrm{MPa}$ but weakened at higher pressures possibly due to the phenomenon known as "over compression". This arises when maximum compressibility has been attained by compaction and further increase in pressure does not lead to an increase in bonding area. If a higher pressure does not result in more plastic deformation, the stored elastic energy would increase because of the additional load applied on the powder as a result of higher pressure. Elastic recovery of particles during decompression then breaks the bonds and compromises tablet strength. The higher the compaction pressure is, the greater is the deterioration/weakening of tabletability, because of the more significant elastic recovery [45].

The differences recorded in the tableting profile of SGS and SMS with respect to the crushing strength and disintegration time can be attributed to the material attributes of both excipients. Due to the high degree of plasticity observed with SMS, tablets containing SMS were observed to have higher crushing strength due to superior bonding area and bonding strength developed during compression. However, disintegration of the tablets (containing SMS) was faster and this correlates with its high swelling capacity which was about 4 times more than that of SGS. Higher values of DER for SMS implies that a better balance of mechanical and disintegrating properties was attained. Hence, the use of SMS as a directly compressible excipient in tablet formulation will not exert a negative effect on tensile strength or disintegrating profile of the tablets [47]. The two co-processed excipients evaluated showed similarities in the tableting profile and functionality and this is consistent with the requirements for an ideal DC excipient. Differences observed in physical and mechanical properties of the excipients can be ascribed to the incorporation of different binders in their formulation.

\section{Conclusion}

This study has revealed that the material and tableting properties of the co-processed excipients developed were consistent with the type of binder used in its formulation. Particle size of the co-processed excipients was influenced by the aggregating ability of the binder used in its preparation with SGS containing gelatin as binder giving rise to composite particles with larger particle diameter. The coprocessed excipient containing microcrystalline cellulose as binder (SMS) demonstrated a higher degree of plasticity during compaction leading to the formation of tablets with better compactibility and tabletability owing to the excellent plastic deformation profile of MCC. Tablets with rapid disintegration time were produced with SMS as a result of the better swelling property of MCC compared to gelatin. The rational selection of a binder in the formulation of co-processed excipient is one critical factor to be considered during its development.

Acknowledgement Yonni Apeji received funding from the Tertiary Education Trust Fund (TETFUND) disbursed through Ahmadu Bello University, Zaria, Nigeria.

Conflict of interest On behalf of all authors, the corresponding author states that there is no conflict of interest. 


\section{References}

1. Thoorens G, Krier F, Leclercq B, Carlin B, Evrard B (2014) Microcrystalline cellulose, a direct compression binder in a quality by design environment-a review. Int J Pharm 473:64-72

2. Goyanes A, Souto C, Martínez-Pacheco R (2011) Co-processed MCC-Eudragit ${ }^{\circ} \mathrm{E}$ excipients for extrusion-spheronization. Eur J Pharm Biopharm 79:658-663. https://doi.org/10.1016/j. ejpb.2011.07.013

3. Nachaegari SK, Bansal AK (2004) Coprocessed excipients for solid dosage forms. Pharm Technol 28(1):52-64

4. Chaheen M, Sanchez-Ballester NM, Bataille B, Yassine A, Belamie E, Sharkawi T (2018) Development of coprocessed chitin-calcium carbonate as multifunctional tablet excipient for direct compression. J Pharm Sci. https://doi.org/10.1016/j. xphs.2018.04.013

5. Apeji YE, Oyi AR, Isah AB, Allagh TS, Modi SR, Bansal AK (2018) Development and optimization of a starch-based co-processed excipient for direct compression using mixture design. AAPS Pharm Sci Tech 18:866-880. https://doi.org/10.1208/s1224 9-017-0887-x

6. Katsuno E, Tahara K, Takeuchi Y, Takeuchi H (2013) Orally disintegrating tablets prepared by a co-processed mixture of micronized crospovidone and mannitol using a ball mill to improve compactibility and tablet stability. Powder Technol 241:60-66. https://doi.org/10.1016/j.powtec.2013.03.008

7. Daraghmeh N, Chowdhry B, Leharne S, Omari M, Badwan A (2015) Co-processed chitin-mannitol as a new excipient for oro-dispersible tablets. Mar Drugs 13:1739-1764. https://doi. org/10.3390/md13041739

8. Chauhan SI, Nathwani SV, Soniwala MM, Chavda JR (2016) Development and characterization of multifunctional directly compressible co-processed excipient by spray drying method. AAPS PharmSciTech. https://doi.org/10.1208/s12249-016-0598-8

9. Rojas J, Kumar V (2011) Comparative evaluation of silicified microcrystalline cellulose II as a direct compression vehicle. Int J Pharm 416:120-128. https://doi.org/10.1016/j.ijpha rm.2011.06.017

10. Rojas J, Buckner I, Kumar V (2012) Co-processed excipients with enhanced direct compression functionality for improved tableting performance. Drug Dev Ind Pharm 38:1159-1170. https:// doi.org/10.3109/03639045.2011.645833

11. Wang S, Li J, Lin X, Feng Y, Kou X, Babu S et al (2015) Novel coprocessed excipients composed of lactose, HPMC, and PVPP for tableting and its application. Int J Pharm 486:370-379. https ://doi.org/10.1016/j.ijpharm.2015.03.069

12. Sharma P, Modi SR, Bansal AK (2015) Co-processing of hydroxypropyl methylcellulose (HPMC) for improved aqueous dispersibility. Int J Pharm 485:348-356

13. Debotton N, Dahan A (2017) Applications of Polymers as Pharmaceutical Excipients in Solid Oral Dosage Forms. Med Res Rev 37:52-97. https://doi.org/10.1002/med

14. Olowosulu AK, Oyi A, Isah AB, Ibrahim MA (2011) Formulation and evaluation of novel coprocessed excipients of maize starch and acacia gum (StarAc) for direct compression tabletting. Int J Pharm Res Innov 2:39-45

15. Apeji $Y E$, Aluga D, Olayemi $O$, Oparaeche $C$, Anyebe $S$, Gamlen $M$ et al (2017) Comparative analysis of co-processed starches developed by three different methods. Br J Pharm. https://doi. org/10.5920/bjpharm.2017.08

16. Adeoye O, Alebiowu G (2014) Flow, packing and compaction properties of novel coprocessed multifunctional directly compressible excipients prepared from tapioca starch and mannitol. Pharm Dev Technol 7450:901-910. https://doi. org/10.3109/10837450.2013.840843
17. Heckel RW (1961) Density-pressure relationships in powder compaction. Trans Metall Soc AIME 221:671-675

18. Walker EE (1923) The properties of powders. Part VI. The compressibility of powders. Trans Faraday Soc 19:73. https://doi. org/10.1039/tf9231900073

19. Sonnergaard JM (2006) Quantification of the compactibility of pharmaceutical powders. Eur J Pharm Biopharm 63:270-277. https://doi.org/10.1016/j.ejpb.2005.10.012

20. British Pharmacopoeia (2013) London. TSO, UK

21. USP29-NF24 (2009) USP monograph: ibuprofen tablets

22. Saha S, Shahiwala AF (2009) Multifunctional coprocessed excipients for improved tabletting performance. Expert Opin Drug Deliv 6:197-208. https://doi.org/10.1517/1742524080 2708978

23. Li Z, Lin X, Shen L, Hong Y, Feng Y (2017) Composite particles based on particle engineering for direct compaction. Int $J$ Pharm 519:272-286

24. Kittipongpatana OS, Kittipongpatana N (2011) Preparation and physicomechanical properties of co-precipitated rice starchcolloidal silicon dioxide. Powder Technol 217:377-382. https:// doi.org/10.1016/j.powtec.2011.10.051

25. Singh I, Birender K, Prateek J (2014) Preparation and characterization of starch-metal silicate co-precipitates-evaluation as tablet superdisintegrant. Polim Med 44:157-166. https://doi. org/10.1046/j.1365-2923.2004.01776.x

26. Mshelia J, Apeji Y, Olayemi O (2015) Powder, compaction and tableting properties of co-processed silicified starch. Br J Pharm Res 6:131-140. https://doi.org/10.9734/BJPR/2015/16139

27. Odeku OA (2005) Assessment of Albizia zygia gum as a binding agent in tablet formulations. Acta Pharm 55:263-276

28. Osei-Yeboah F, Feng Y, Sun CC (2014) Evolution of structure and properties of granules containing microcrystalline cellulose and polyvinylpyrrolidone during high-shear wet granulation. J Pharm Sci 103:207-215. https://doi.org/10.1002/jps.23776

29. Charoenthai N, Sanga-ngam TSP (2018) Use of modified tapioca starches as pharmaceutical excipients. Pharm Sci Asia 45:195-204

30. Odeku OA (2013) Potentials of tropical starches as pharmaceutical excipients: a review. Starch/Staerke 65:89-106. https://doi. org/10.1002/star.201200076

31. El-Sakhawy M, Hassan ML (2007) Physical and mechanical properties of microcrystalline cellulose prepared from agricultural residues. Carbohydr Polym 67:1-10

32. Hou HAO, Sun CC (2008) Quantifying effects of particulate properties on powder flow properties using a ring shear tester. J Pharm Sci 97:4030-4039. https://doi.org/10.1002/jps

33. Singh S, Rao RKV, Venugopal K, Manikandan R (2002) Alteration in dissolution characteristics of gelatin-containing formulations: a review of the problem, test methods, and solutions. Pharm Technol 26(4):36-58

34. Sinko PJ, Singh Y (eds) (2011) Pharmaceutical polymers. Martins' Physics Pharmacy Pharmacuticles Science. Wolters Kluwer/Lippincott Williams \& Wilkins, Philadelphia, pp 492-515

35. Ogunjimi AT, Alebiowu G (2013) Flow and consolidation properties of neem gum coprocessed with two pharmaceutical excipients. Powder Technol 246:187-192. https://doi.org/10.1016/j. powtec.2013.04.051

36. Desai PM, Liew CV, Heng PWS (2016) Review of disintegrants and the disintegration phenomena. J Pharm Sci 105:2545-2555. https://doi.org/10.1016/j.xphs.2015.12.019

37. Jain S (1999) Mechanical properties of powders for compaction and tableting: an overview. Pharm Sci Technol Today 2:20-31. https://doi.org/10.1016/S1461-5347(98)00111-4

38. Ghori MU, Conway B (2016) Powder compaction: compression properties of cellulose ethers. Br J Pharm 1:19-29. https://doi. org/10.5920/bjpharm.2016.09 
39. Chattoraj S, Sun CC (2018) Crystal and particle engineering strategies for improving powder compression and flow properties to enable continuous tablet manufacturing by direct compression. J Pharm Sci 107:968-974. https://doi.org/10.1016/j. xphs.2017.11.023

40. Zhou D, Qiu Y (2010) Understanding material properties in pharmaceutical product development and manufacturing: powder flow and mechanical properties. J Valid Technol 16(2):65-77

41. Zhang J, Wu CY, Pan X, Wu C (2017) On identification of critical material attributes for compression behaviour of pharmaceutical diluent powders. Materials (Basel) 10:1-16. https://doi. org/10.3390/ma10070845

42. Lamešic D, Planinšek O, Lavric Z, Ilic I (2017) Spherical agglomerates of lactose with enhanced mechanical properties. Int J Pharm 516:247-257

43. Yadav JA, Khomane KS, Modi SR, Ugale B, Yadav RN, Nagaraja CM et al (2017) Correlating single crystal structure, nanomechanical, and bulk compaction behavior of febuxostat polymorphs. Mol Pharm 14:866-874. https://doi.org/10.1021/acs.molpharmac eut.6b01075
44. Joiris E, Di Martino P, Berneron C, Guyot-Hermann A, Guyot J (1998) Compression behavior of orthorhombic paracetamol. Pharm Res 15:1122-1130

45. Sun CC (2011) Decoding powder tabletability: roles of particle adhesion and plasticity. J Adhes Sci Technol 25:483-499. https ://doi.org/10.1163/016942410X525678

46. Khomane KS, More PK, Raghavendra G, Bansal AK (2013) Molecular understanding of the compaction behavior of indomethacin polymorphs. Mol Pharm 10:631-639. https://doi. org/10.1021/mp300390m

47. Adeoye O, Alebiowu G (2014) Dimensionless quantities in the evaluation of novel composite disintegrants. J Drug Deliv Sci Technol 24:222-228

Publisher's Note Springer Nature remains neutral with regard to jurisdictional claims in published maps and institutional affiliations. 